\title{
Relapse Patterns and Outcomes Following Recurrence of Endometrial Cancer in Northern Thai Women
}

\author{
Phakdee Kaewpangchan ${ }^{1}$, Chalong Cheewakriangkrai ${ }^{2 *}$
}

\begin{abstract}
Background: The aim of this study was to analyze the patterns of relapse and survival outcomes in Northern Thai women with recurrent endometrial cancer (EC). Materials and Methods: Medical records were abstracted from EC patients who underwent primary surgery from 1999 to 2012. Data on clinicopathologic variables, sites of first recurrence, time to relapse of disease, and overall survival (OS) was analyzed. Associations between the clinicopathological variables and the rates of disease recurrence were determined. Results: Among 1,204 reviewed records, 42 eligible patients were identified with recurrent disease. The median age was 55 years and the median follow-up time was 26.0 months. The median times to recurrence (TTR) after completion of the initial treatment in the group of local relapse (LR) and distant/combined sites of recurrence (DCSR) was 6.6 (95\% CI=4.6 to 8.6 months) and 16.9 months $(95 \% \mathrm{CI}=5.6$ to 28.2 months), respectively ( $p=0.36)$. The 2-year survival and 3-year survival probability in the group of $L R$ was $54.2 \%(95 \% \mathrm{CI}=27.2$ to $81.3 \%)$ and $34.7 \%(95 \%$ CI=9.2 to 60.2\%), compared to $50.4 \%(95 \% \mathrm{CI}=41.1$ to $59.7 \%)$ and $42.1 \%(95 \% \mathrm{CI}=\mathbf{2 4 . 1}$ to $60.1 \%)$ for those with DCSR. Distant recurrence was the most frequent pattern of relapse. Overall survival was not significantly different in patients with local relapse when compared to those with DCSR $(p=0.69)$. Conclusions: Patients with recurrence of EC after primary treatment had a worse prognosis and clinical aggressiveness. LR and DCSR occurred most during the first three years. The common sites of relapses were vaginal cuff, pelvis, and lungs. No significant clinicopathological predictor for survival outcomes was identified.
\end{abstract}

Keywords: Endometrial cancer - recurrence - relapse - Northern Thailand

Asian Pac J Cancer Prev, 16 (9), 3861-3866

\section{Introduction}

Endometrial cancer is the most common female genital tract malignancy, and the fourth most common cancer in North American women, accounting for about $6 \%$ of new cancer cases and 3\% of cancer deaths per year (Siegel et al., 2014). Risk factors such as exogenous hormone use and obesity and tumor characteristics including histologic subtype vary by race (Panggid et al., 2010; Jamison et al., 2013; Demiral et al., 2014). The five year survival rate was $95 \%, 68 \%$, or $17 \%$, if the cancer was diagnosed at a local, regional, or distant stage, respectively (Siegel et al., 2014). Most patients were diagnosed as early stage endometrial cancer that appeared to be confined to the uterus. Recurrence after primary treatment remains one of the major clinical problems in women with high-risk early stage and advanced disease (Setakornnukul et al., 2014). The recurrence rate has been reported and varied in between 10\%-60\% (Morrow et al., 1991; Kaminski et al., 1992; Podczaski et al., 1992; Berchuck et al., 1995). Although much is known on the treatment and prognostic factors for endometrial cancer, there are only few studies on patterns of relapse and long-term outcomes after relapse occurs. This study was aimed to review the patterns of relapse and survival outcomes in Northern Thai women with recurrent endometrial cancer

\section{Materials and Methods}

After Research Ethics Committee approval was obtained, the medical records of all Northern Thai women with endometrial cancer who underwent primary surgery with or without adjuvant radiotherapy and/ or chemotherapy at Chiang Mai University Hospital from January, 1999 through December, 2012 were retrospectively reviewed. All cases with recurrent tumors were identified, forming the study population. Exclusion criteria included co-existing malignancies, immunodeficiency or severe underlying illness that limited the standards of treatment, and initial surgical treatment at other institutions. Patients with at least a total abdominal hysterectomy (TAH) and bilateral salpingo-oophorectomy (BSO) for low-risk stage I were included in the study and were not differentiated from those with high-risk stage I-IV undergoing TAH, BSO, omentectomy, and pelvic and/or paraaortic lymph node dissection. Surgical staging 
Phakdee Kaewpangchan and Chalong Cheewakriangkrai was based on the 2009 FIGO staging system. Patients with a high risk of relapse received adjuvant therapy after surgery, whereas those with an intermediate risk were at the physician's discretion. Patients with occult cervical stromal invasion treated with simple hysterectomy were given postoperative adjuvant radiation therapy (RT), including external beam RT with or without intravaginal brachytherapy as vaginal stump boost. Moreover, adjuvant chemotherapy was recommended for positive cytology, adnexal metastasis, or tumors of nonendometrioid histology (clear cell, papillary serous, or undifferentiated carcinoma). Adjuvant chemotherapy consisted of cisplatin of $60 \mathrm{mg} / \mathrm{m}^{2}$ or carboplatin AUC 5 in combination with paclitaxel of $175 \mathrm{mg} / \mathrm{m}^{2}$ or Adriamycin $60 \mathrm{mg} /$ $\mathrm{m}^{2}$ every three weeks for a total of six cycles. Patients with lymph node or distant metastases received adjuvant chemotherapy (cisplatin of $60 \mathrm{mg} / \mathrm{m}^{2}$ or carboplatin AUC5 in combination with paclitaxel of $175 \mathrm{mg} / \mathrm{m}^{2}$ or Adriamycin $60 \mathrm{mg} / \mathrm{m}^{2}$ every three weeks) for six cycles, tumor directed RT was added depending on the tumor site and patient's performance status as a sequential/ sandwich chemotherapy and RT. Extended field RT up to L1 or T12 level was applied for patients with paraaortic node metastases. After completion of treatment, all patients were followed every three months in the first year, every four months in the second year and every six months thereafter. Recurrence was defined by clinical, radiological, or imaging evidence and/or histological confirmation as appropriate. Recurrences were classified as local if they were detected in the pelvis or vagina and
Table 1. The Clinicopathological Characteristics of 42 Patients with Recurrent Endometrial Cancer

\begin{tabular}{lc}
\hline Characteristics & No. of pts $(\%)$ \\
\hline Age (years), median & $55(40-78)$ \\
$\quad \leq 50$ & $7(16.7)$ \\
$>50$ & $35(83.3)$ \\
Body mass index $(\mathrm{kg} / \mathrm{m} 2)$, median & $23.9(16-43)$ \\
$\quad<25$ & $24(57.1)$ \\
$\geq 25$ & $18(42.9)$ \\
FIGO (2009) stage & \\
I & $12(28.6)$ \\
II & $4(9.5)$ \\
III & $20(47.6)$ \\
IV & $6(14.3)$ \\
Tumor grade & \\
1 & $15(35.7)$ \\
2 & $15(35.7)$ \\
3 & $12(28.6)$ \\
Histologic type & \\
Endometrioid & $31(73.8)$ \\
Non-endometrioid & $11(26.2)$ \\
Lymphovascular space involvement & \\
Negative & $14(33.3)$ \\
Positive & $28(66.7)$ \\
Depth of myometrial invasion & \\
$<1 / 2$ & $18(42.9)$ \\
$\geq 1 / 2$ & $24(57.1)$ \\
First site of recurrence & $13(30.9)$ \\
Local & $22(52.4)$ \\
Distant & $7(16.7)$ \\
Combined & \\
\hline &
\end{tabular}

Table 2. Analysis of Clinicopathological Variables on Site of Recurrence among Endometrial Cancer Patients

\begin{tabular}{|c|c|c|c|c|c|}
\hline Variable & Local & $\begin{array}{l}\text { Site of recurrence } \\
\text { Distant/Combined }\end{array}$ & Odd ratio & $95 \% \mathrm{CI}$ & p-value \\
\hline Age (years) & & & & $0.35-9.93$ & 0.66 \\
\hline$\leq 50$ & 3 & 4 & 1.67 & & \\
\hline$>50$ & 10 & 25 & 0.89 & & \\
\hline Body mass index $\left(\mathrm{kg} / \mathrm{m}^{2}\right)$ & & & & $0.21-3.04$ & 0.75 \\
\hline$<25$ & 7 & 17 & 0.91 & & \\
\hline$\geq 25$ & 6 & 12 & 1.13 & & \\
\hline Lymphovascular space involvement & & & & $0.21-3.44$ & 1.00 \\
\hline Yes & 9 & 19 & 1.06 & & \\
\hline No & 4 & 10 & 0.89 & & \\
\hline Myometrial invasion & & & & $0.20-2.93$ & 0.70 \\
\hline$>1 / 2$ & 8 & 16 & 1.12 & & \\
\hline$\leq 1 / 2$ & 5 & 13 & 0.86 & & \\
\hline Tumor grade & & & & $0.04-1.20$ & 0.09 \\
\hline 1 & 2 & 13 & 0.34 & & \\
\hline 2 or more & 11 & 16 & 1.53 & & \\
\hline Histologic type & & & & $0.10-1.75$ & 0.27 \\
\hline Endometrioid & 8 & 23 & 0.78 & & \\
\hline Non-endometrioid & 5 & 6 & 1.86 & & \\
\hline FIGO stage & & & & $0.08-1.63$ & 0.3 \\
\hline I-II & 3 & 13 & 0.52 & & \\
\hline III-IV & 10 & 16 & 1.39 & & \\
\hline Pelvic LN involvement & & & & $0.07-1.10$ & 0.06 \\
\hline Yes & 8 & 9 & 1.98 & & \\
\hline No & 5 & 20 & 0.56 & & \\
\hline Para-aortic LN involvement & & & & $0.14-3.48$ & 0.69 \\
\hline Yes & 3 & 5 & 1.34 & & \\
\hline No & 10 & 24 & 0.93 & & \\
\hline
\end{tabular}


as distant if they were detected in extrapelvic locations. Time to recurrence (TTR) was defined as a period of time to detect any recurrence after completion of primary treatment. Time to progression was calculated from a period of time since diagnosis until disease progression. Subsequent sites of progressive disease after the first relapse were not included in the data analysis.

\section{Statistical analysis}

Overall survival (OS) duration was calculated from the date of diagnosis to the date of death or the last follow-up visit. Time to recurrence (TTR) was calculated from the date of completion of the primary treatment to the date of disease relapse. Progression-free survival (PFS) duration was calculated from the date of first diagnosis to the date of disease recurrence. Data analyses were performed with the SPSS software package for Windows (version 17.0; SPSS Inc., Chicago, IL). Association between the clinicopathological variables and the rates of disease recurrences were compared using Pearson's chi-square or Fisher's exact test, and likelihood ratio methods. Survival was calculated and survival curves were generated using the Kaplan-Meier method. The comparison of survival difference was performed by log-rank test. A probability value of $<0.05$ was considered statistically significant.

\section{Results}

From the one thousand two hundred four patients that underwent primary surgery for endometrial cancer during the study period, 60 patients with relapse of disease were identified. The overall recurrence rate after the primary treatment was five percent $(60 / 1204)$. Of these 60 patients, 18 were excluded. The reasons for exclusion were as follows: dual primary malignancies $(n=3)$; severe underlying disease that could limit the standard of treatment $(n=4)$; pathologic information not available $(n=3)$; initial surgical treatment at other institutions $(n=4)$; and lost to follow-up $(n=4)$. Forty-two patients were included in the final analysis.

Clinical and pathologic characteristics of the study population are presented in Table 1 . The median age was 55 years (range, 40-78) and median body mass index (BMI) was $23.9 \mathrm{~kg} / \mathrm{m} 2$ (range, 15.6-42.5). Of the 42 patients, $16(38.1 \%)$ had FIGO stage I or II disease, and $26(61.9 \%)$ had FIGO stage III or IV disease. The common histologic type was endometrioid histology that was observed in $31(73.8 \%)$ patients. Grade II and III tumor was found in $15(35.7 \%)$ and 12 (28.6\%) patients, respectively. The majority of tumors showed lymphovascular space involvement (66.7\%) and depth of myometrial invasion was more than half $(57.1 \%)$.

Distant relapse was found in $10(62 \%)$ and $12(46 \%)$ of patients with Stages I-II and Stages III-IV, respectively. However, local failure was more common in patients with Stages III-IV compared to Stages I-II (Table 2). The common sites of relapse were vagina, pelvic cavity, and lungs. Among 42 patients with recurrent endometrial cancer, sites of recurrence were found as follows: 15 $(35.7 \%)$ vaginal cuff, $12(28.6 \%)$ pelvic cavity, 11 (26.2\%) lungs, five (11.9\%) abdomen, five (11.9\%) paraaortic lymph nodes, three $(7.1 \%)$ liver, two $(4.8 \%)$ supraclavicular lymph nodes, two $(4.8 \%)$ inguinal lymph nodes, two $(4.8 \%)$ bone, one $(2.4 \%)$ brain, one $(2.4 \%)$ bladder, and one (2.4\%) chest wall. Seven patients $(16.7 \%)$ were present with more than one site of relapse. The effect of age, BMI, lymphovascular space involvement (LVSI), depth of myometrial invasion, tumor grade, histologic type, pelvic and paraaortic lymph node involvement, and FIGO stage was examined between the group of patients that had local and distant/combined sites of recurrence (Table 2). We found that age $>50$ years was more frequent in both those who had local (10 of 13,76.9\%) and distant/ combined ( 25 of $29,86.2 \%$ ) sites of relapse. Risk factors such as LVSI, deep myometrial invasion, high-grade tumor, and advanced stage were also more frequent in the patients with distant/combined sites of relapse but there was not statistically significant. The median follow-up time was 26 months (range, 3.4-127.0). Median time to recurrence (TTR) after completion of initial treatment in the group of local relapse and distant/combined sites of relapse was $6.6(95 \% \mathrm{CI}=4.6$ to 8.6 months $)$ and 16.9 months (95\% CI=5.6 to 28.2 months), respectively ( $\mathrm{p}=0.36$ ). For patients who had local relapse, the 2-year survival and 3 -year survival probability was $54.2 \%(95 \% \mathrm{CI}=27.2$ to $81.3 \%$ ) and $34.7 \%(95 \% \mathrm{CI}=9.2$ to $60.2 \%)$, compared

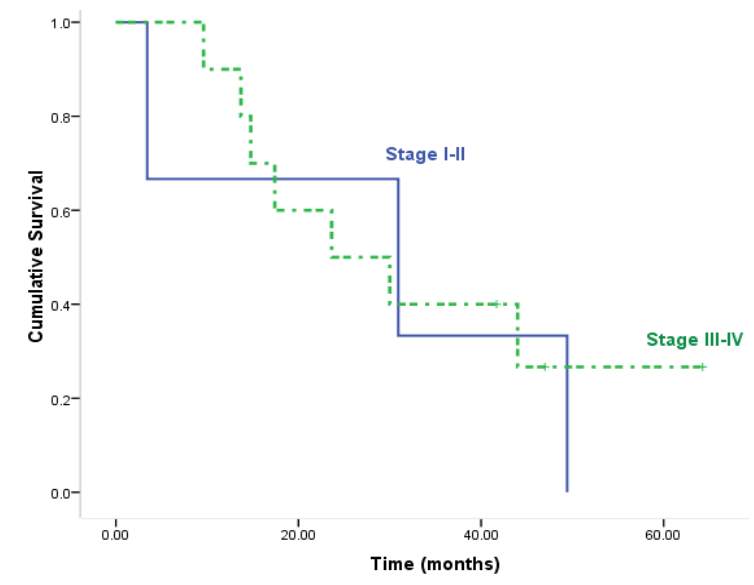

Figure 1. Comparison of Kaplan-Meier Survival Curves in Patients with Local Relapses According to FIGO Stage I-II versus FIGO Stage III-IV, $p=0.19$

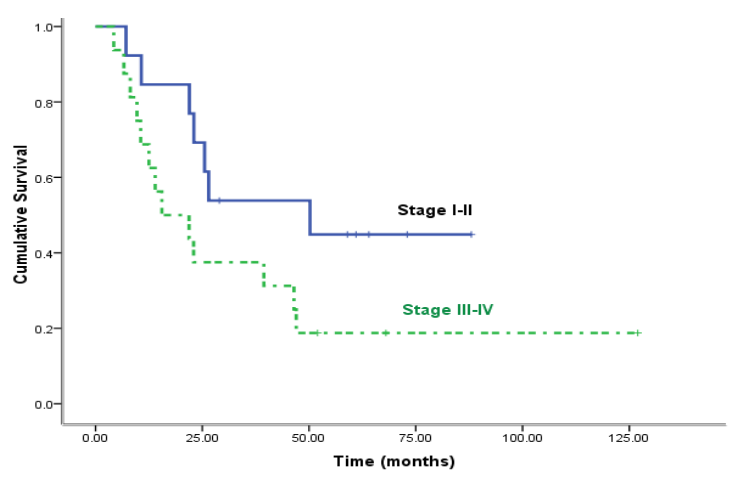

Figure 2. Comparison of Kaplan-Meier Survival Curves in Patients with Distant/ Combined Sites of Relapses According to FIGO Stage I-II versus FIGO Stage III-IV, $p=0.19$ 
Phakdee Kaewpangchan and Chalong Cheewakriangkrai

Table 3. Analysis of Clinicopathological Variables on Overall Survival among Endometrial Cancer Patients with Recurrence

\begin{tabular}{|c|c|c|c|c|c|}
\hline Variable & "No. of patients" & No. of deaths & Hazard ratio & $95 \% \mathrm{CI}$ & p-value \\
\hline Age (years) & & & & $0.11-1.72$ & 0.24 \\
\hline$\leq 50$ & 6 & 4 & 0.43 & & \\
\hline$>50$ & 34 & 26 & 1.00 & & \\
\hline Body mass index $\left(\mathrm{kg} / \mathrm{m}^{2}\right)$ & & & & $0.66-3.37$ & 0.34 \\
\hline$<25$ & 23 & 16 & 1.49 & & \\
\hline$\geq 25$ & 17 & 12 & 1.00 & & \\
\hline Lymphovascular space involvement & & & & $0.38-2.36$ & 0.91 \\
\hline Yes & 14 & 9 & 0.95 & & \\
\hline No & 26 & 21 & 1.00 & & \\
\hline Myometrial invasion & & & & $0.42-2.57$ & 0.93 \\
\hline$>1 / 2$ & 18 & 13 & 1.04 & & \\
\hline$\leq 1 / 2$ & 22 & 17 & 1.00 & & \\
\hline Tumor grade & & & & $0.23-1.62$ & 0.32 \\
\hline 1 & 15 & 9 & 0.61 & & \\
\hline 2 or more & 25 & 21 & 1.00 & & \\
\hline Histologic type & & & & $0.18-1.16$ & 0.09 \\
\hline Endometrioid & 29 & 21 & 0.10 & & \\
\hline Non-endometrioid & 11 & 9 & 1.00 & & \\
\hline FIGO stage & & & & $0.18-1.56$ & 0.25 \\
\hline I-II & 16 & 10 & 0.53 & & \\
\hline III-IV & 24 & 20 & 1.00 & & \\
\hline Pelvic LN involvement & & & & $0.45-5.29$ & 0.49 \\
\hline Yes & 24 & 18 & 1.55 & & \\
\hline No & 16 & 12 & 1.00 & & \\
\hline Para-aortic LN involvement & & & & $0.27-3.64$ & 0.98 \\
\hline Yes & 32 & 25 & 0.98 & & \\
\hline No & 8 & 5 & 1.00 & & \\
\hline Site of recurrence & & & & $0.40-2.52$ & 0.99 \\
\hline Local & 13 & 10 & 0.99 & & \\
\hline Distant/ combined & 27 & 20 & 1.00 & & \\
\hline
\end{tabular}

to $50.4 \%(95 \% \mathrm{CI}=41.1$ to $59.7 \%)$ and $42.1 \%(95 \% \mathrm{CI}=$ 24.1 to $60.1 \%$ ) for those with distant/combined sites of recurrence. Overall survival was not significantly different in patients with local relapse when compared to those with distant/combined sites of relapse $(\mathrm{p}=0.69)$. When stratified by FIGO stage, the overall survival in patients with early-stage (stage I and II) endometrial cancer who had local and distant/combined sites of recurrence was longer than those with advanced stage (stage III and IV) but was not statistically significant (Figure 1 and Figure 2). Comparison of clinicopathological variables between the group of local relapse and distant/combined sites of recurrence was demonstrated in Table 2. There were no clinicopathological factors found significantly to be predictive for survival in patients with recurrent endometrial cancer (Table 3).

\section{Discussion}

Surgical therapy is a mainstay and primary treatment of endometrial cancer. A comprehensive surgery allows for accurate information on disease extent. FIGO endometrial cancer staging is based on surgical pathology which includes a hysterectomy, bilateral salpingooophorectomy and pelvic/para-aortic lymph node dissection with pelvic washings and, for poor histologic types, an omental biopsy (Cragun et al., 2005). Patients with Grade1 and Grade 2 endometrial tumors, less than $50 \%$ myometrium invasion, and tumor of two $\mathrm{cm}$ or less seem to be at low risk for recurrence and may not require a surgical lymphadenectomy.(Burker et al., 2014) Management and adjuvant treatment after surgery depends upon a patient's risk factors for recurrence. Adjuvant radiation for certain Stage I or II endometrial cancers reduces the locoregional recurrence rate but does not affect overall survival (Krusun et al., 2014; Setakornnukul et al., 2014). Chemotherapy and radiation therapy used in combination may offer superior outcomes compared with single-modality treatment in those with advanced-stage disease (Burke et al., 2014). However, recurrences of endometrial cancer after initial treatment remain one of the major clinical problems. Most reports in the literature focus on recurrent disease after Stage I and II primary disease because the majority of patients with endometrial cancer usually present with early stage disease without extrauterine spread. Recurrence developed in about $10-20 \%$ of patients whose disease was confined to the uterus and about $40-60 \%$ of those who had adnexal and lymph node metastasis (Morrow et al., 1991; Podczaski et al., 1992; Kaminski et al. 1992; Berchuck et al., 1995). In our series the recurrence rate was five percent. This may differ from the results of previous studies due to the different period of available chemotherapy, paclitaxel and carboplatin in combination, which were commonly used after the year 1995. Most failures in patients with Stage I and II primary disease were at distant sites beyond the pelvis that could represent some occult micrometastases which could not be covered by local surgery and radiotherapy 
at the beginning. In a retrospective review, Sohaib et al. (2007) demonstrated that $87 \%$ of all recurrences occurred by the third year after primary treatment compared with $93 \%$ in the present study. A median time to local recurrence of 11.5 months and to distant recurrence of 20.5 months, this compares with 6.6 and 10.6 months in our study, respectively. These results support the need for close follow-up during the first three years after primary treatment. The number of patients presenting with local relapse and distant/combined sites of recurrence were seen in $31 \%$ and $69 \%$ compared with $35 \%$ and $65 \%$ in the report from Sohaib et al. (2007). These results were quite similar to the study from Agboola et al. (1997) in 50 patients with recurrent endometrial cancer that demonstrated local disease in $38 \%$ and distant disease in $62 \%$. The common sites of relapse were found at the vaginal cuff, pelvic cavity, and lungs. Distant recurrence was the most frequent pattern of relapse in the present study and found scattered in lungs, abdomen, paraaortic and supraclavicular lymph nodes, inguinal lymph nodes, liver, bone, brain, bladder, and chest wall. However, simultaneous local and distant recurrence was also seen in $17 \%$ among the patients with recurrent endometrial cancer. These findings could add important data on distant hematogenous spread cells of primary tumor and support the role of systemic chemotherapy. About $62 \%$ of our patients had advanced FIGO stage (Stage III and Stage IV) and the chance to have either local relapse or distant/combined sites of recurrence was relatively higher compared with those who had early FIGO stage (Stages I and II) but the data did not show any statistical significance. When local relapse occurred radiation therapy can yield an effective local control, whereas, chemotherapy still has some modest activity to control advanced disease or distant recurrence. Adjuvant radiation for certain Stage I or II endometrial carcinomas reduces the locoregional recurrence rate but does not affect overall survival (Burke et al, 2014). The use of combination chemotherapy for patients with Stage III and IV endometrial cancer has been reported and the 3-year overall survival rates varied between $56 \%$ and $75 \%$ (Hidaka et al., 2006; Sovak et al., 2006). However, if local failure occurred the overall survival of the patients did not seem to be different from those with distant failure. The 2-year survival rate was about $54 \%$ in those who had local relapse compared with $50 \%$ in those who had distant or simultaneous local and distant recurrence. In this study, $77 \%$ of the patients with first episode of local recurrence and $69 \%$ with distant spread died of their disease. This may be due to the current limitations of available chemotherapy regimen and second-or third-line therapy in these groups of patients. Shortly after detection of recurrences and initiation of secondary treatment, the disease progression was documented frequently in a certain period of time. The median time to progression was 11.5 months. The following clinicopathological factors: patient's age at primary surgery, BMI, depth of myometrial invasion, LVSI, tumor grade, histologic type, FIGO stage, pelvic and paraaortic lymph node status, and site of recurrence were not found to significantly affect overall survival of the patients after recurrence of disease. In contrast, in a study by Huang et al. (2014) on prognostic factors after treatment failure of endometrial cancer, initial Stage II to IV, Type II histology, positive peritoneal cytology, and recurrence at multiple sites were significantly associated with poor survival after recurrence.

There are some limitations to the present study due to the small number of patients and the rarity of recurrence of disease in endometrial cancer. However, most such cases were treated and followed regularly at a gynecologic oncology center.

In conclusion, patients with recurrence of endometrial cancer after primary treatment had a worse prognosis and clinical aggressiveness. Both local relapse and distant or combined sites of recurrences occurred most during the first three years. The common site of distant relapses was lungs, whereas, the local relapses were commonly found at vaginal cuff and pelvis. There were no predictive clinicopathological factors for survival outcomes to be found.

\section{References}

Agboola OO, Grunfeld E, Coyle D, Perry GA (1997). Costs and benefits of routine follow-up after curative treatment for endometrial cancer. CMAJ, 157, 879-86.

Berchuck A, Anspach C, Evans AC, et al (1995). Postsurgical surveillance of patients with FIGO stage I/II endometrial adenocarcinoma. Gynecol Oncol, 59, 20-4.

Burke WM, Orr J, Leitao M, et al (2014). Endometrial cancer: a review and current management strategies: part II. Gynecol Oncol, 134, 393-402.

Burke WM, Orr J, Leitao M, et al (2014). Endometrial cancer: a review and current management strategies: part I. Gynecol, 134, 385-92.

Cragun JM, Havrilesky LJ, Calingaert B, et al (2005). Retrospective analysis of selective lymphadenectomy in apparent early-stage endometrial cancer. J Clin Oncol, 23, 3668-75.

Demiral S, Beyzadeoglu M, Sager O, et al (2014). Evaluation of treatment outcomes of early-stage endometrial cancer radiotherapy: a single center experience. Asian Pac J Cancer Prev, 15, 9599-602.

Hidaka T, Nakamura T, Shima T, Yuki H, Saito S (2006). Paclitaxel/carboplatin versus cyclophosphamide/adriamycin/ cisplatin as postoperative adjuvant chemotherapy for advanced endometrial adenocarcinoma. J Obstet Gynaecol Res, 32, 330-7.

Huang HJ, Tang YH, Chou HH, et al (2014). Treatment failure in endometrial carcinoma. Int J Gynecol Cancer, 24, 885-93.

Jamison PM, Noone AM, Ries LA, Lee NC, Edwards BK (2013). Trends in endometrial cancer incidence by race and histology with a correction for the prevalence of hysterectomy, SEER 1992 to 2008. Cancer Epidemiol Biomarkers Prev, 22, 233-41.

Krusun S, Pesee M, Rasio W, et al (2014). Survival rate of early stage endometrioid adenocarcinoma of endometrium treated at Srinagarind Hospital. Asian Pac J Cancer Prev, 15, 2217-20.

Morrow CP, Bundy BN, Kurman RJ, et al (1991). Relationship between surgical-pathological risk factors and outcome in clinical stage I and II carcinoma of the endometrium: a Gynecologic Oncology Group study. Gynecol Oncol, 40, 55-65.

Panggid KC, Cheewakriangkrai C, Khunamornpong S, Siriaunkgul S (2010). Factors related to recurrence in non-obese women with endometrial endometrioid 
Phakdee Kaewpangchan and Chalong Cheewakriangkrai adenocarcinoma. J Obstet Gynaecol Res, 36, 1044-8.

Podczaski E, Kaminski P, Gurski K, et al (1992). Detection and patterns of treatment failure in 300 consecutive cases of "early" endometrial cancer after primary surgery. Gynecol Oncol, 47, 323-7.

Setakornnukul J, Petsuksiri J, Wanglikitkoon S, et al (2014). Long term outcomes of patients with endometrial carcinoma treated with radiation - Siriraj Hospital experience. Asian Pac J Cancer Prev, 15, 2279-85.

Siegel RJ, Ma J, Zou Z, Jemal A (2014). Cancer statistics, 2014. CA Cancer J Clin, 64, 9-29.

Sohaib SA, Houghton SL, Meroni R, et al (2007). Recurrent endometrial cancer: patterns of recurrent disease and assessment of prognosis. Clin Radiol, 62, 28-34.

Sovak MA, Hensley ML, Dupont J, et al (2006). Paclitaxel and carboplatin in the adjuvant treatment of patients with highrisk stage III and IV endometrial cancer: a retrospective study. Gynecol Oncol, 103, 451-7. 